\title{
Risk and Protective Factors for Arthritis Status and Severity
}

\author{
Masaru Teramoto*1, Fred Breukelman², Ferdinando A. Gatto ${ }^{2}$ and Sheniz Moonie ${ }^{3}$ \\ ${ }^{1}$ Health Sciences, Drexel University, Philadelphia, PA, USA; ${ }^{2}$ Delaware Health and Social Services, Dover, DE, USA; ${ }^{3}$ University of \\ Nevada, Las Vegas, Las Vegas, NV, USA
}

\section{Objective}

To examine how cigarette smoking, alcohol consumption, obesity, and physical activity are associated with the risk and severity of arthritis among adults living in Delaware.

\section{Introduction}

Research has indicated several risk and protective factors for arthritis, including cigarette smoking, alcohol consumption, obesity, and physical activity (1-4). However, it is not well understood how all these factors interact to increase/decrease the risk of arthritis.

\section{Methods}

Data from the 2009 Delaware Behavioral Risk Factor Surveillance System (BRFSS) were analyzed in the current study. Potential risk and protective factors for arthritis status and severity examined in this study included: smoking status, alcohol consumption, weight status, and physical activity level. Weighted percentages were calculated for the risk and protective factors by arthritis status and activity limitation due to arthritis/joint symptoms, and were analyzed using a RaoScott $\chi 2$ test. A logistic regression analysis was performed to determine an odds ratio (OR) while adjusting for gender, age, race/ethnicity, and education.

\section{Results}

Adults living in Delaware self-reporting arthritis were more likely to be former and current smokers than those without self-reported arthritis $(\mathrm{p}<0.001, \mathrm{OR}=1.64-1.70)$. Moderate and heavy alcohol drinking was associated with lower prevalence and severity of arthritis $(\mathrm{p}<0.001, \mathrm{OR}=0.45-0.74)$. There was a significant relationship between obesity and arthritis status or activity limitation due to arthritis/joint symptoms $(\mathrm{p}<0.01, \mathrm{OR}=1.62-2.14)$. Furthermore, people with arthritis having activity limitation due to arthritis/joint symptoms were more likely to not meet the current physical activity recommendations (5) $(\mathrm{p}=0.013, \mathrm{OR}=1.49)$

\section{Conclusions}

Cigarette smoking, alcohol consumption, obesity, and physical activity are all associated with the prevalence and severity of arthritis. It is possible that smoking and obesity have a negative impact on the risk and severity of arthritis, whereas alcohol consumption and physical activity may reduce its risk and severity. Further research, including prospective cohort studies, is necessary to determine the true absolute risk of developing arthritis, so that we can design the effective prevention strategies.
Table 1. Risk and protective factors by arthritis status and severity

\begin{tabular}{|c|c|c|c|c|c|c|}
\hline & \multicolumn{3}{|c|}{$\begin{array}{l}\text { Arthritis status }(\mathrm{N}= \\
\text { 3969) }\end{array}$} & \multicolumn{3}{|c|}{$\begin{array}{l}\text { Activity limitation due to arthritis/joint } \\
\text { symptoms }(\mathrm{N}=1512)\end{array}$} \\
\hline & Yes & No & & Yes & No & \\
\hline $\begin{array}{l}\text { Potential risk/protective } \\
\text { factor }\end{array}$ & $\begin{array}{l}27.5 \\
(0.9)\end{array}$ & $\begin{array}{l}72.5 \\
(0.9)\end{array}$ & $p$-value* & $39.1(1.7)$ & $60.9(1.7)$ & $\mathrm{p}$-value \\
\hline Smoking status & & & $<0.001$ & & & 0.382 \\
\hline Non-smoker & $\begin{array}{l}42.1 \\
(1.8)\end{array}$ & $\begin{array}{l}57.7 \\
(1.5)\end{array}$ & & $40.6(2.6)$ & $43.0(2.4)$ & \\
\hline Former smoker & $\begin{array}{l}39.3 \\
(1.7)\end{array}$ & $\begin{array}{l}24.1 \\
(1.2)\end{array}$ & & $38.4(2.6)$ & $39.9(2.3)$ & \\
\hline Current smoker & $\begin{array}{l}18.6 \\
(1.4)\end{array}$ & $\begin{array}{l}18.2 \\
(1.2)\end{array}$ & & $21.0(2.2)$ & $17.1(1.9)$ & \\
\hline Alcohol consumption & & & $<0.001$ & & & $<0.001$ \\
\hline No drinking & $\begin{array}{l}52.4 \\
(1.8)\end{array}$ & $\begin{array}{l}38.8 \\
(1.5)\end{array}$ & & $61.8(2.6)$ & $46.5(2.4)$ & \\
\hline Moderate drinking & $\begin{array}{l}27.2 \\
(1.6) \\
\end{array}$ & $\begin{array}{l}34.3 \\
(1.5) \\
\end{array}$ & & $24.9(2.3)$ & $28.5(2.2)$ & \\
\hline Heavy drinking & $\begin{array}{l}20.4 \\
(1.6)\end{array}$ & $\begin{array}{l}26.9 \\
(1.4)\end{array}$ & & $13.3(1.8)$ & $25.0(2.2)$ & \\
\hline $\begin{array}{l}\text { Weight status (body mass } \\
\text { index }{ }^{\text {a) }}\end{array}$ & & & $<0.001$ & & & 0.008 \\
\hline Not overweight/obese & $\begin{array}{l}26.3 \\
(1.6)\end{array}$ & $\begin{array}{l}41.0 \\
(1.6)\end{array}$ & & $21.0(2.2)$ & $29.7(2.3)$ & \\
\hline Overweight & $\begin{array}{l}36.3 \\
(1.7)\end{array}$ & $\begin{array}{l}35.3 \\
(1.5)\end{array}$ & & $36.0(2.6)$ & $36.5(2.3)$ & \\
\hline Obese & $\begin{array}{l}37.4 \\
(1.8)\end{array}$ & $\begin{array}{l}23.7 \\
(1.2)\end{array}$ & & $43.0(2.6)$ & $33.8(2.4)$ & \\
\hline $\begin{array}{l}\text { Recommended physical } \\
\text { activity }^{\mathrm{b}}\end{array}$ & & & $<0.001$ & & & 0.013 \\
\hline Yes & $\begin{array}{l}43.7 \\
(1.8) \\
\end{array}$ & $\begin{array}{l}53.7 \\
(1.6) \\
\end{array}$ & & $38.2(2.7)$ & $47.3(2.5)$ & \\
\hline No & $\begin{array}{l}56.3 \\
(1.8)\end{array}$ & $\begin{array}{l}46.3 \\
(1.6)\end{array}$ & & $61.8(2.7)$ & $52.7(2.5)$ & \\
\hline
\end{tabular}

Notes: Values given as \% (SE).

${ }^{a}$ Not overweight/obese: $<25.0 \mathrm{~kg} / \mathrm{m} 2$, Overweight: $25.0-29.9 \mathrm{~kg} / \mathrm{m} 2$, Obese $\geq 30 \mathrm{~kg} / \mathrm{m} 2$.

${ }^{\mathrm{b}}$ Moderate physical activity for $\geq 30$ minutes/day on $\geq 5$ days/week, or vigorous physical activity for $\geq 20$ minutes/day on $\geq 3$ days/week (5).

*Rao-Scott $\chi 2$ test.

\section{Keywords}

alcohol; smoking; arthritis; Behavioral Risk Factor Surveillance

\section{References}

1. Albano SA, Santana-Sahagun E, Weisman MH. Cigarette smoking and rheumatoid arthritis. Semin Arthritis Rheum 2001; 31:146-159.

2. Maxwell JR, Gowers IR, Moore DJ, et al. Alcohol consumption is inversely associated with risk and severity of rheumatoid arthritis. Rheumatology 2010; 49:2140-2146.

3. Anandacoomarasamy A, Caterson I, Sambrook P, et al. The impact of obesity on the musculoskeletal system. Int J Obes 2008; 32:211-222.

4. Manninen P, Riihimaki H, Heliovaara M, et al. Physical exercise and risk of severe knee osteoarthritis requiring arthroplasty. Rheumatology $2001 ; 40: 432-437$.

5. Haskell WL, Lee IM, Pate RR, et al. Physical activity and public health: updated recommendation for adults from the American College of Sports Medicine and the American Heart Association. Circulation 2007; 116:1081-1093.

*Masaru Teramoto

E-mail: Masaru.Teramoto@drexel.edu 